\title{
Intraocular perfluorodecalin and silicone oil tamponade (double filling) in the management of complicated retinal detachment: functional and anatomical outcomes using small-gauge surgery
}

\author{
Emma Clara Zanzottera ${ }^{1}$ (D) Alessandro Marchese ${ }^{2} \cdot$ Francesco Bandello $^{2} \cdot$ Michele Coppola $^{1}$
}

Received: 26 April 2021 / Revised: 16 August 2021 / Accepted: 23 August 2021 / Published online: 22 September 2021

( ) The Author(s), under exclusive licence to Springer-Verlag GmbH Germany, part of Springer Nature 2021

\begin{abstract}
Purpose To describe the functional and anatomical results of complicated retinal detachment (RD) treated with small-gauge pars plana vitrectomy (PPV) and combined perfluorodecalin and polydimethylsiloxane tamponade (double filling, DF).

Methods Retrospective analysis of consecutive patients with complex RD (severe proliferative vitreoretinopathy, inferior/ posterior/giant retinal tears, and traumatic detachments) treated with small-gauge PPV, membrane peeling, and DF at the Department of Ophthalmology at San Gerardo Hospital, Monza, Italy. Main outcome measures included best-corrected visual acuity (BCVA), rates of retinal reattachment, and complications.

Results This study included 15 patients with a median follow-up (FU) of 6 months (range 1-22). Three patients with early retinal redetachment under tamponade and FU shorter than 3 months were excluded from the final functional analysis, but they were considered anatomical failure. At the last examination, BCVA improved in 50\% of patients and remained stable in $25 \%$ of patients and anatomical success was achieved in $73 \%$ of eyes, $64 \%$ of them without any endotamponade. Three eyes had retinal redetachment after perfluorodecalin/silicone oil exchange because of diffuse proliferative vitreoretinopathy (PVR) and required reoperation to achieve retinal attachment. In eyes with anatomical success, macular pucker was the most frequent long-term complication (27\%).

Conclusion In the management of complex RD, small-gauge pars plana vitrectomy, and double filling endotamponade using wideangle viewing systems was a well-tolerated and effective technique to preserve visual acuity and achieve anatomical success.
\end{abstract}

\section{Key messages}

- Complicated rhegmatogenous retinal detachment (RD) represents a significant cause of visual morbidity and surgical failure.

- Patients with complicated RD could be effectively treated with small gauge PPV.

- In the management of complicate RD, small gauge PPV and combined perfluorodecalin and polydimethylsiloxane tamponade (double filling, DF) improved or stabilized vision in $75 \%$ of eyes and achieved retinal attachment in $73 \%$ of eyes.

Keywords Double filling $\cdot$ Pars plana vitrectomy $\cdot$ Proliferative vitreoretinopathy $\cdot$ Retinal detachment

Emma Clara Zanzottera

e.zanzottera@asst-monza.it

1 Department of Ophthalmology, Department of Ophthalmology, San Gerardo Hospital, via Gian Battista Pergolesi 33, 20900 Monza, Italy

2 Department of Ophthalmology, IRCCS San Raffaele Scientific Institute, Milan, Italy

\section{Introduction}

Complex rhegmatogenous retinal detachment (RD) still represents a significant cause of visual morbidity and surgical failure. Different risk factors for surgical failure have been identified throughout the years, including the 
presence of proliferative vitreoretinopathy (PVR), multiple and large retinal tears, severe ocular trauma, and late surgery [1-7].

Intraocular tamponades have been used at the end of the surgery for RD to provide support to the reattached retina before a permanent retinopexy has been obtained, either by laser or cryopexy. In some of the most complex cases of RD, an optimal retinopexy can be difficult to achieve. In these cases, various therapeutic strategies have been used, including the injection of two different tamponade agents together. This technique, known as "double filling" (DF), consists of the use of both perfluorocarbon liquids (PFCL) and silicone oil to temporarily stabilize the retina before exchanging with silicone oil.

In this study, we report our experience using smallgauge pars plana vitrectomy (PPV), "DF technique" and wide-angle viewing systems to treat patients with complicated RD.

\section{Materials and methods}

We reviewed the clinical data from all the consecutive patients with complicated RD (25 cases) who underwent 25- or 23-G PPV and DF tamponade (combined perfluorodecalin and polydimethylsiloxane) between December 2018 and February 2021 at the Department of Ophthalmology at San Gerardo Hospital, Monza, Italy. The study adhered to the tenets of the Declaration of Helsinki and received the approval of the local institutional review board.

Each surgery was performed by an experienced vitreoretinal surgeon (M.C. and M.S.). Briefly, after lensectomy in selected cases, the surgeon performed a small-gauge PPV, epiretinal membrane peeling, and relaxing peripheral retinotomies (according to the surgeon's clinical judgment) to mobilize the retina. Retinal reattachment was achieved with the help of purified perfluorocarbon liquid (perfluorodecalin, $\mathrm{C}_{10} \mathrm{~F}_{18}$ ) and laser photocoagulation of retinal breaks and retinotomy. At the end of the surgery, the vitreous cavity was filled with perfluorodecalin $(60 \%$ of vitreous chamber) and polydimethylsiloxane (PDMS, $1000 \mathrm{cSt}, 40 \%$ of vitreous chamber). All surgeries were performed using the Alcon CONSTELLATION vitrectomy machine, a wide-angle noncontact viewing system and a contact macular lens when necessary. The surgical approach, including instrument gauge and tamponade agent, was based on the surgeon's preference at the time of surgery. No patients received a scleral buckle in addition to PPV and DF because, in most cases, PVR was extended to retroequatorial space and the retina was poorly mobile. Surgeries were performed using general anesthesia. All patients maintained postoperative supine positioning and remained hospitalized for 1 week until a PFCL/silicone oil exchange was performed using local anesthesia. Intraocular pressure was controlled with oral acetazolamide. Bestcorrected visual acuities (BCVA) were recorded using a Snellen chart.

\section{Results}

This study enrolled 15 eyes of 15 patients. Eyes missing detailed data or achieving good anatomical results with less than 3 months FU were excluded. Tractional RDs in proliferative diabetic retinopathy were excluded. Pathology at preoperative examination is described in Table 1, while patients' demographics, preoperative functional, and anatomical characteristics are summarized in Table 2.

All patients were Caucasian. The average age of patients at surgery was 56 years (range 18-85) and $80 \%$ were men. Three patients presented with high myopia; one eye was amblyopic. Eight eyes were phakic, six were pseudophakic, and one was aphakic as a result of penetrating injury. Seven patients (47\%) had undergone previous surgical attempts at repair of RD (PPV with gas or silicone oil tamponade or scleral buckling, 10 days to 8 months before DF surgery); one patient underwent previous successful PPV for macular pucker and one patient had complicated macular surgery two months before RD surgery.

\section{Preoperative functional records}

Preoperatively, the BCVA was count finger (CF), hand motion (HM), or light perception (LP) in 13 eyes (86\%), 20/400 in 1 eye, and 20/50 in 1 eye. All but one patient was referred to the department within a few days to one month from the first or recurrent onset of vision loss; one patient reported a progressive vision loss during the last 8 months after non-penetrating trauma.

\section{Preoperative anatomical records}

All patients presented with macula-off $\mathrm{RD}$, severe and inferior PVR (grades C-D, Retina Society Classification, defined intraoperatively); four patients showed PVR at the posterior

Table 1 Pathology at preoperative examination

15 RD with severe, inferior PVR and/or inferior retinal tears

$6 \mathrm{RD}$ with posterior or inferior giant tears

4 RD secondary to ocular trauma (3 penetrating trauma, 1 blunt trauma)

2 Retinal and choroidal detachment

$R D$, retinal detachment; $P V R$, proliferative vitreoretinopathy 


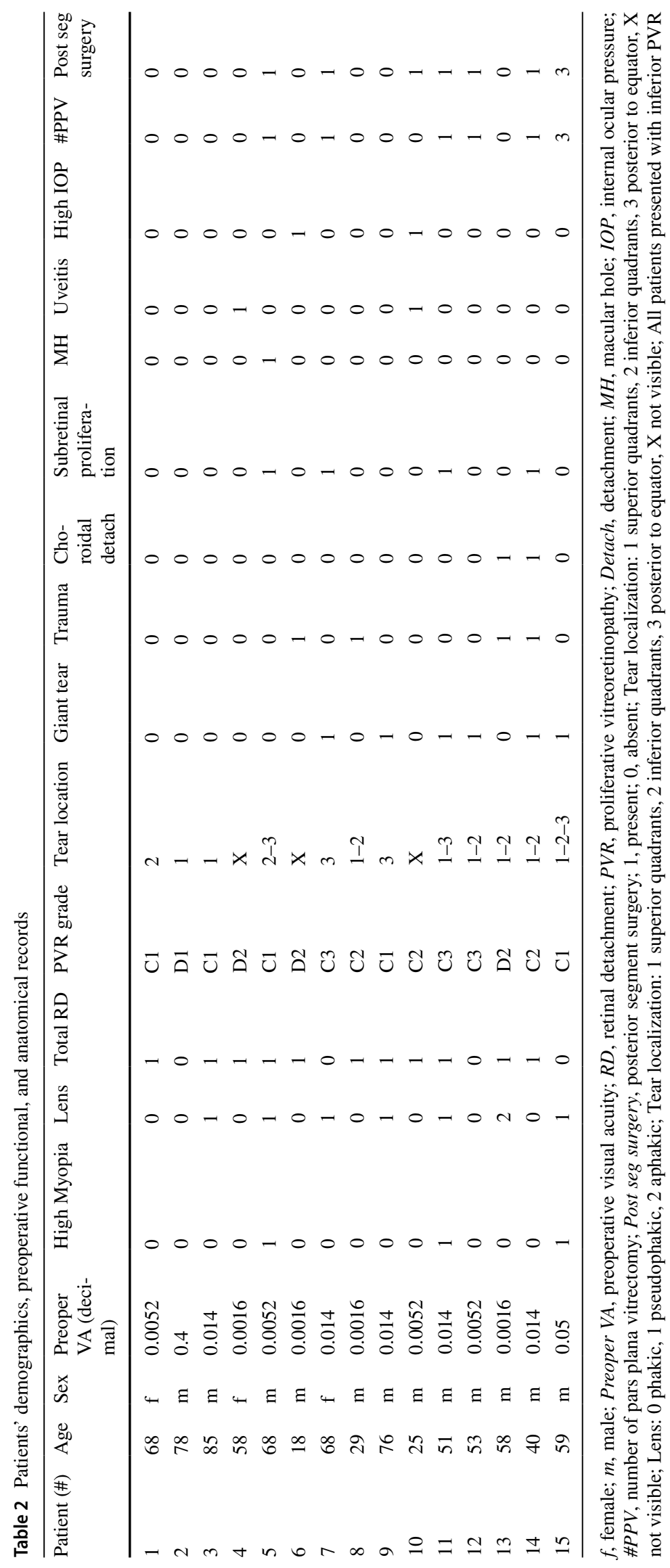


pole at presentation. Eleven eyes $(73 \%)$ had total RD, 7 eyes (47\%) showed inferior retinal breaks, 6 eyes (40\%) reported posterior or inferior giant tears, 3 eyes (15\%) had penetrating trauma, and 2 eyes (13\%) presented with retinal and choroidal detachment. Four eyes (27\%) had subretinal proliferation. Four patients (27\%) presented with vitreous hemorrhage. Full-thickness macular hole occurred in one case, uveitis in 2 cases. In three eyes the location of retinal tear was not recognized; seven cases $(47 \%)$ presented with multiple retinal tears located in different quadrants (superior, inferior, and posterior to the equator). Two young patients used therapy for ocular hypertension associated with uveitis and posttraumatic iris new vessels. One patient showed recurrent $\mathrm{RD}$ and branch retinal vein occlusion.

\section{Surgical records}

Patients were managed with limbal lensectomy with implantation of intraocular lens ( 7 eyes, capsulotomy was performed according to the surgeon's clinical judgment), small-gauge three-port PPV, or revision of prior vitrectomy (13 eyes received 25-G PPV, 2 eyes 23G PPV), epiretinal membrane dissection and peeling, relaxing circumferential retinotomies (10 eyes, extended from one quadrant to $360^{\circ}$, mostly inferiorly and larger than two quadrants), internal tamponade with perfluorodecalin (60\% of vitreous cavity) and PDMS (40\% of vitreous cavity), endolaser photocoagulation, and postoperative supine positioning until PFCL removal. One patient (case no. 7) required removal of blood, PDMS, and PFCL located in subretinal space from previous surgery; a trauma case received a combined corneal transplant. Intraoperative complete retinal reattachment was achieved in all cases. Meantime of ocular surgery was $1 \mathrm{~h}$ and $40 \mathrm{~min}$. Seven days after the first surgery, a PFCL/silicone oil exchange was performed; 14 eyes injected PDMS, 1 eye injected densiron (polydimetylsiloxane $5000 \mathrm{cSt}$ and perfluorohexyloctane). Patients' details regarding PFCL/PDMS surgery and PFCL/ silicone oil exchange are illustrated in Table 3.

\section{Results at last follow-up}

The median follow-up (FU) was 6 months (range 1-22, mean FU 9,4 months). Clinical records are reported in Table 4. Three patients (cases nos. 7, 12, and 13) with early redetachment under PDMS or densiron were not further treated because of poor prognosis and they were considered exclusively in the anatomical analysis section of this study.

\section{Functional results}

At the final FU visit (at 4 to 22 months), visual acuity improved in 6 eyes (50\%), remained stable in 3 eyes (25\%) and decreased in 3 eyes (25\%). BCVA was CF/HM/LP in 4 eyes (33\%), 20/200 or less in 4 eyes (33\%), and $20 / 50$ or more in 3 eyes (25\%). One eye turned blind. Two patients with the decreased vision developed PVR at the posterior pole and inferior recurrent PVR-associated RD within 1 month after PFCL/PDMS exchange; one patient lost vision because of severe hypotony. Among traumatic RD, one patient gained vision, one stabilized, and one lost vision. Fourteen patients were pseudophakic at the final FU, one patient remained aphakic.

Table 3 Surgical records

\begin{tabular}{|c|c|c|c|c|c|c|}
\hline Patient (\#) & Age & Sex & $\begin{array}{l}\text { Relaxing } \\
\text { retinotomy }\end{array}$ & $\begin{array}{l}\text { Duration of PFCL/ } \\
\text { PDMS surgery (min) }\end{array}$ & $\begin{array}{l}\text { PFCL/PDMS surgery } \\
\text { combined with } \\
\text { phaco + iol }\end{array}$ & $\begin{array}{l}\text { PFCL/silicone } \\
\text { oil exchange }\end{array}$ \\
\hline 1 & 68 & $\mathrm{f}$ & 1 & 60 & 1 & pdms \\
\hline 2 & 78 & $\mathrm{~m}$ & 1 & 65 & 1 & pdms \\
\hline 3 & 85 & $\mathrm{~m}$ & 0 & 85 & 0 & pdms \\
\hline 4 & 58 & $\mathrm{f}$ & 1 & 60 & 1 & pdms \\
\hline 5 & 68 & $\mathrm{~m}$ & 0 & 110 & 0 & pdms \\
\hline 6 & 18 & $\mathrm{~m}$ & 1 & 120 & 1 & pdms \\
\hline 7 & 68 & $\mathrm{f}$ & 1 & 130 & 0 & pdms \\
\hline 8 & 29 & $\mathrm{~m}$ & 0 & 100 & 1 & pdms \\
\hline 9 & 76 & $\mathrm{~m}$ & 0 & 100 & 0 & pdms \\
\hline 10 & 25 & $\mathrm{~m}$ & 1 & 120 & 0 & pdms \\
\hline 11 & 51 & $\mathrm{~m}$ & 1 & 125 & 0 & pdms \\
\hline 12 & 53 & $\mathrm{~m}$ & 1 & 95 & 1 & pdms \\
\hline 13 & 58 & $\mathrm{~m}$ & 1 & 120 & 0 & densiron \\
\hline 14 & 40 & $\mathrm{~m}$ & 1 & 100 & 1 & pdms \\
\hline 15 & 59 & $\mathrm{~m}$ & 0 & 70 & 0 & pdms \\
\hline
\end{tabular}

Relaxing retinotomy (1, yes; 0 , no); min, minutes; PFCL/PDMS surgery combined with phaco + iol (1), not combined with phaco + iol (0); pdms, polydimethylsiloxane 
Table 4 Postoperative functional and anatomical records at last follow-up

\begin{tabular}{lllllll}
\hline Patient (\#) & Age & Sex & $\begin{array}{l}\text { Postoper VA at last } \\
\text { FU (decimal) }\end{array}$ & $\begin{array}{l}\text { Postoper retinal } \\
\text { status at last FU }\end{array}$ & $\begin{array}{l}\text { Last FU } \\
\text { (months) }\end{array}$ & $\begin{array}{l}\text { Tamponade at } \\
\text { last FU }\end{array}$ \\
\hline 1 & 68 & $\mathrm{f}$ & 0.4 & 0 & 6 & - \\
2 & 78 & $\mathrm{~m}$ & 0.05 & 0 & 8.5 & - \\
3 & 85 & $\mathrm{~m}$ & 0.0052 & 1 & 4 & - \\
4 & 58 & $\mathrm{f}$ & 0.05 & 0 & 21 & - \\
5 & 68 & $\mathrm{~m}$ & 0.014 & 0 & 22 & pdms \\
6 & 18 & $\mathrm{~m}$ & 0.0016 & 0 & 4 & pdms \\
7 & 68 & $\mathrm{f}$ & 0.0052 & 1 & 2.5 & pdms \\
8 & 29 & $\mathrm{~m}$ & 0.5 & 0 & 10 & - \\
9 & 76 & $\mathrm{~m}$ & 0.1 & 0 & 16 & - \\
10 & 25 & $\mathrm{~m}$ & 0.5 & 0 & 20 & - \\
11 & 51 & $\mathrm{~m}$ & 0.014 & 0 & 12 & pdms \\
12 & 53 & $\mathrm{~m}$ & 0.05 & 1 & 1 & densiron \\
13 & 58 & $\mathrm{~m}$ & 0.0052 & 1 & 1.5 & densiron \\
14 & 40 & $\mathrm{~m}$ & 0 & 0 & 6 & pdms \\
15 & 59 & $\mathrm{~m}$ & 0.05 & 0 & 6 & - \\
\hline
\end{tabular}

$V A$, visual acuity; $F U$, follow-up; pdms, polydimethylsiloxane; Postoper, postoperative; retinal status al last $F U$ : attached retina (0), detached retina (1)

\section{Anatomical results}

At the final examination (at 1 to 22 months), eleven patients $(73 \%)$ had a retinal attachment and four patients $(27 \%)$ presented with early (within 1 month) recurrence of severe, inferior, or diffuse PVR and RD after PFCL/silicone oil exchange.

In the group with final retinal success, seven patients removed any tamponade (median FU 10 months, range 6-21), and four patients required PDMS permanently (median FU 9 months, range 4-22). Among those cases, 3 eyes with open-funnel RD at first presentation showed retinal redetachment after 1 to 6 months from PFCL/PDMS exchange, because of posterior and diffuse PVR; two of them were managed with second PPV, membrane peeling, enlargement of extended peripheral retinotomies, and densiron endotamponade to obtain final anatomical success; the other one, who reported a chronic RD at first examination, underwent membrane peeling, second PFCL/PDMS surgery, and persistent PDMS tamponade. Three eyes $(27 \%)$ received additional laser treatment during FU.

Seventy-five percent of traumatic RDs achieved anatomical success, but one of them completely lost visual function because of severe hypotony. The youngest patient with long-lasting RD and iris rubeosis secondary to nonpenetrating ocular trauma maintained light perception BCVA and flat retina at 4 months FU.

Among patients with early retinal attachment, four eyes removed silicone oil after 2 to 5 months; the other two cases removed silicone oil after 7 and 9 months because of COVID-19-related organization issues.
In the group with early retinal redetachment (at 1 to 4 months), two patients (cases \# 3, 12) underwent a second and last PPV and membrane peeling (additional retinotomy in one case) without anatomical success; the other two patients (cases \# 7, 13) with more advanced pathology (severe PVR, vitreous hemorrhage, choroidal detachment, and penetrating trauma) did not receive any further surgery because of poor prognosis.

The major postoperative complication was recurrent RD due to contracture of residual and/or recurrent epiretinal membranes. In eyes achieving anatomical success, the development of macular pucker was the most frequent complication (27\%). One penetrating trauma case developed severe hypotony; patients with intraocular pressure higher than $21 \mathrm{mmHg}$ were effectively treated with topical drugs. No signs of significant persistent intraocular inflammation were observed during FU; one penetrating trauma eye showed PFCL emulsification in the anterior chamber associated with mild inflammation and without corneal endothelial decompensation; no retained subretinal PFCL was recorded.

\section{Discussion}

Complex RD characterized by severe and inferior PVR, posterior and inferior giant tears, penetrating trauma, or combined rhegmatogenous and choroidal detachment shows a high risk of anatomical failure. Primary surgical goals are treatment of both rhegmatogenous and tractional components and effective intra- and postoperative 
endotamponade, sealing retinal breaks, and displacing fluid from retinal surface in order to limit epiretinal reproliferation.

Previous studies found small-gauge PPV had similar or better outcomes to $20 \mathrm{G}$ instrumentation in complicated RD [8,9]; the advent of wide-angle viewing systems allowed a more comprehensive and peripheral intraoperative examination of the retina, helping the surgeon to achieve more effective maneuvers and exchanges in the management of RD. All those components are included in our surgical retinal approach (M.IL.A.N., Machine/Microincision, ILlumination, Accessories, Non-contact) cited at previous scientific meetings.

Despite these surgical improvements, at present, still no endotamponade shows simultaneous optimal management of both the superior and inferior retina in complex RD $[5,10,11]$. Perfluorocarbon liquids, thanks to the specific gravity higher-than-water, act as effective intraoperative adjuvants to stabilize the detached retina [12] and they even reduced PVR severity postoperatively [13]. Previous studies showed clinical safety of purified PFCLs for postoperative application extended over a week [14-19]. In complicated RD, different groups of clinicians found good ocular tolerance and efficacy of combined PFCL and PDMS ("double filling," DF) endotamponade for variable postoperative timing [20-23].

In our series, perfluorodecalin was used to tamponade the retinal tears at the posterior pole and inferior retina in the first 7 postoperative days and reduce compartmentalization of retinal pigment epithelium cells and cytokines on the retinal surface at those locations minimizing postoperative cell reproliferation and recurrent RD; PDMS stabilized PFCL bubble maintaining its effectiveness on the retina, and preventing PFCL emulsification and migration to the anterior chamber [22, 24, 25].

Analyzing the clinical outcomes of this surgery in our series, BCVA at the most recent visit improved in $50 \%$ of patients and remained stable in $25 \%$ of patients (Table 4); anatomical success was achieved in $73 \%$ of eyes, $64 \%$ of them without any endotamponade (Table 4). Three eyes had retinal redetachment after PFCL/PDMS exchange because of diffuse PVR and required another surgery to achieve final retinal attachment.

Due to the differences in preoperative pathology, surgical procedures, and a number of additional surgeries, it's difficult to compare our results with previously reported surgical outcomes. However, using small-gauge instrumentation and short-term DF endotamponade, our rate of overall successful retinal attachment at the end of FU was similar to the outcomes of other studies which managed complex RD cases, as initial or repeat surgery, with postoperative short or long-term tamponade with different PFCLs combined with PDMS [14, 16, 21-23]. Based on those clinical reports, the intraocular use of perfluorodecalin for 7 days was discussed with the patient prior to surgery.

The retrospective nature of our study, the small sample and the variable preoperative pathology, and FU in our series limit the final analysis to a descriptive approach.

In our cohort, the preoperative BCVA and the presence of giant or posterior retinal tears did not directly correlate with final visual acuity. All patients with thick subretinal proliferation at the posterior pole achieved worse visual outcomes (BCVA $<0.05)$ according to the extent and severity of the preoperative macular disease; $77 \%$ of eyes without subretinal PVR obtained BCVA $\geq 0.05$. The grade of PVR at baseline (Table 2) was not related with final BCVA; therefore, even if PVR and its severity are known risk factors for RD [6, 26, 27], the grade of PVR seems not to correlate with visual outcome [26, 27]. Indeed, among eyes that required relaxing retinotomies because of most difficult anatomic conditions, $57 \%$ of eyes achieved visual acuity equal to or better than 0.05 . Other studies found that in complex RD, the size of retinotomy did not correlate with functional results [28-30]; in those cases, the visual outcome was more influenced by the posterior retina status.

Regarding the anatomical outcomes of our series, the preoperative BCVA did not directly correlate with sustained retinal attachment, limiting its value as a prognostic factor for anatomical outcome. All patients with high myopia or macular hole achieved retinal attachment with or without endotamponade ( $F U \geq 6$ months). All but one patient with subretinal membranes achieved retinal attachment (FU $\geq 6$ months); therefore, subretinal proliferation might be a poor prognostic factor for the visual outcome (if it is located at the posterior pole) but it doesn't seem to be a risk factor for redetachment, as Hanneken et al. found [26]. Seventy-three percent of patients with inferior PVR at baseline achieved retinal attachment. Among patients with more severe PVR, at final FU, one eye did not obtain retinal attachment and the others received additional surgery to achieve anatomical success; the rate of retinal reattachment correlated with the preoperative PVR grade. Eighty percent of patients with posterior tear and $67 \%$ of patients with giant tear maintained retinal attachment at 6 months or later; 33\% of eyes with giant tear showed early recurrent RD under endotamponade. Overall, these results suggest that the DF technique might contribute to good anatomical outcomes in those patients. Among eyes that required relaxing retinotomies, $70 \%$ of eyes achieved retinal attachment at final examination (FU $\geq 4$ months). The correlation between exposure of retinal pigment epithelium in the areas of the retinotomies and rate of reproliferation and recurrent $\mathrm{RD}$ is debated [25, 29, 31]; size of retinotomies 
and retinectomies did not correlate with worse anatomic results in previous studies $[29,30]$.

It is difficult to correlate the recurrence of epiretinal membranes to the preoperative features or to an inflammatory reaction to the endotamponade. However, during FU, we have not seen perfluorodecalin emulsification or persistent intraocular inflammation. For instance, in uveitic patients, good functional and anatomical outcomes were achieved at 20 months of FU. Moreover, perfluorodecalin caused significantly less toxic damage in comparison to perfluorooctane [32].

Considering the DF technique as the first RD surgery, mean BCVA changed from 0.06 preoperative to 0.14 postoperative; considering DF technique as a second surgery, mean BCVA changed from 0.02 to 0.10 . The patient with the highest number of previous PPV for recurrent RD maintained visual acuity equal to 0.05 . Whether PPV and DF surgery was used as the first approach or as the second approach, final retinal attachment rates were similar $(75 \%$ and $71 \%$ of cases, respectively). Therefore, DF surgery might be an efficient procedure also as secondary surgery.

The lack of a control group prevents definite conclusions regarding the advantages of DF in respect to other endotamponades. However, in our single-center retrospective study of patients with complex retinal detachment, small-gauge vitrectomy and "double filling technique" was a well-tolerated procedure which improved or stabilized vision in $75 \%$ of eyes and achieved retinal attachment in $73 \%$ of eyes.

Acknowledgements We thank Maurizio Sborgia, MD, for his expert vitreoretinal surgery and Michal Post, MD, ESASO fellow at San Gerardo Hospital for assistance in analyzing ophthalmic data of the patients in the study.

Author contribution Emma Clara Zanzottera: conceptualization, data collection, and writing; Alessandro Marchese: writing and editing; Francesco Bandello: supervision; Michele Coppola: conceptualization, surgery, and writing.

Data availability Data are available upon request to the corresponding authors.

\section{Declarations}

Ethics approval The study received the approval of the San Gerardo ASST Monza IRB.

Consent to participate Patients signed written consent at the time of enrollment to be included in observational clinical research studies.

Consent for publication Patients signed written consent at the time of enrollment to be included in observational clinical research studies. No images featuring patients or identifiable details have been used.

Conflict of interest The authors declare no competing interests.

\section{References}

1. Machemer R (1977) Massive periretinal proliferation: a logical approach to therapy. Trans Am Ophthalmol Soc 75:556-586

2. Treister G, Moisseiev J (1983) Combined pars plana vitrectomy and retinal surgery in massive periretinal proliferation. Ann Ophthalmol 15(7):670-671, 674-675

3. Ryan SJ (1985) The pathophysiology of proliferative vitreoretinopathy in its management. Am J Ophthalmol 100(1):188-193. https://doi.org/10.1016/s0002-9394(14)75004-4

4. Lewis H, Aaberg TM (1991) Causes of failure after repeat vitreoretinal surgery for recurrent proliferative vitreoretinopathy. Am J Ophthalmol 111(1):15-19. https://doi.org/10.1016/s00029394(14)76890-4

5. Pastor JC (1998) Proliferative vitreoretinopathy: an overview. Surv Ophthalmol 43(1):3-18. https://doi.org/10.1016/s00396257(98)00023-x

6. Williamson TH, Lee EJ, Shunmugam M (2014) Characteristics of rhegmatogenous retinal detachment and their relationship to success rates of surgery. Retina 34(7):1421-1427. https://doi.org/ 10.1097/iae.0000000000000094

7. Mudhar HS (2020) A brief review of the histopathology of proliferative vitreoretinopathy (PVR). Eye (Lond) 34(2):246-250. https://doi.org/10.1038/s41433-019-0724-4

8. Storey PP, Ter-Zakarian A, Philander SA, Olmos de Koo L, George M, Humayun MS, Rodger DC, Ameri H (2018) Visual and anatomical outcomes after diabetic traction and traction-rhegmatogenous retinal detachment repair. Retina 38(10):1913-1919. https://doi.org/10.1097/iae.0000000000001793

9. Narala R, Nassiri N, Kim C, Mehregan C, Padidam S, Abrams GW (2018) Outcomes of repeat pars plana vitrectomy after failed surgery for proliferative vitreoretinopathy. Retina 38(Suppl 1):S49-S59. https://doi.org/10.1097/iae.0000000000002000

10. Yamamoto S, Takeuchi S (2000) Silicone oil and fluorosilicone. Semin Ophthalmol 15(1):15-24. https://doi.org/10.3109/08820 530009037847

11. Foster WJ (2008) Vitreous substitutes. Expert Rev Ophthalmol 3(2):211-218. https://doi.org/10.1586/17469899.3.2.211

12. Chang S, Reppucci V, Zimmerman NJ, Heinemann MH, Coleman DJ (1989) Perfluorocarbon liquids in the management of traumatic retinal detachments. Ophthalmology 96 (6):785-791; discussion 791-782. https://doi.org/10.1016/s0161-6420(89)32812-0

13. Stolba U, Binder S, Velikay M, Datlinger P, Wedrich A (1995) Use of perfluorocarbon liquids in proliferative vitreoretinopathy: results and complications. Br J Ophthalmol 79(12):1106-1110. https://doi.org/10.1136/bjo.79.12.1106

14. Bottoni F, Sborgia M, Arpa P, De Casa N, Bertazzi E, Monticelli M, De Molfetta V (1993) Perfluorocarbon liquids as postoperative short-term vitreous substitutes in complicated retinal detachment. Graefes Arch Clin Exp Ophthalmol 231(11):619-628. https://doi. org/10.1007/bf00921955

15. Kobuch K, Menz IH, Hoerauf H, Dresp JH, Gabel VP (2001) New substances for intraocular tamponades: perfluorocarbon liquids, hydrofluorocarbon liquids and hydrofluorocarbon-oligomers in vitreoretinal surgery. Graefes Arch Clin Exp Ophthalmol 239(9):635-642. https://doi.org/10.1007/s004170100330

16. Bottoni F, Bailo G, Arpa P, Prussiani A, Monticelli M, de Molfetta V (1994) Management of giant retinal tears using perfluorodecalin as a postoperative short-term vitreoretinal tamponade: a long-term follow-up study. Ophthalmic Surg 25(6):365-373

17. Sirimaharaj M, Balachandran C, Chan WC, Hunyor AP, Chang AA, Gregory-Roberts J, Hunyor AB, Playfair TJ (2005) Vitrectomy with short term postoperative tamponade using perfluorocarbon liquid for giant retinal tears. Br J Ophthalmol 89(9):11761179. https://doi.org/10.1136/bjo.2004.065409 
18. Rofail M, Lee LR (2005) Perfluoro-n-octane as a postoperative vitreoretinal tamponade in the management of giant retinal tears. Retina 25(7):897-901. https://doi.org/10.1097/00006982-20051 0000-00013

19. Gremillion CM Jr, Peyman GA, Liu KR, Naguib KS (1990) Fluorosilicone oil in the treatment of retinal detachment. Br J Ophthalmol 74(11):643-646. https://doi.org/10.1136/bjo.74.11.643

20. Bottoni F, Arpa P, Vinciguerra P, Zenoni S, De Molfetta V (1992) Combined silicone and fluorosilicone oil tamponade (double filling) in the management of complicated retinal detachment. Ophthalmologica 204(2):77-81. https://doi.org/10.1159/000310273

21. Rizzo S, Genovesi-Ebert F, Belting C, Foltran F, Gandolfo E, Lesnoni G, Dell'omo E, Zenoni S, Azzolini M, De Molfetta V (2005) Long-term vitreous replacement with perfluorohexyloctane and silicone oil: preliminary reports of a multicentric study. Ophthalmologica 219(3):147-153. https://doi.org/10.1159/000085247

22. Rizzo S, Genovesi-Ebert F, Belting C (2006) The combined use of perfluorohexyloctane (F6H8) and silicone oil as an intraocular tamponade in the treatment of severe retinal detachment. Graefes Arch Clin Exp Ophthalmol 244(6):709-716. https://doi.org/10. 1007/s00417-006-0317-3

23. Zenoni S, Romano MR, Palmieri S, Comi N, Fiorentini E, Fontana P (2011) Ocular tolerance and efficacy of short-term tamponade with double filling of polydimethyloxane and perfluoro-n-octane. Clin Ophthalmol 5:443-449. https://doi.org/10.2147/opth.s17315

24. Herbert E, Stappler T, Wetterqvist C, Williams R, Wong D (2004) Tamponade properties of double-filling with perfluorohexyloctane and silicone oil in a model eye chamber. Graefes Arch Clin Exp Ophthalmol 242(3):250-254. https://doi.org/10.1007/ s00417-003-0830-6

25. Kirchhof B, Wong D, Van Meurs J, Hilgers RD, Macek M, Lois N, Schrage NF (2002) Use of perfluorohexyloctane as a longterm internal tamponade agent in complicated retinal detachment surgery. Am J Ophthalmol 133(1):95-101. https://doi.org/10. 1016/s0002-9394(01)01295-8

26. Hanneken AM, Michels RG (1988) Vitrectomy and scleral buckling methods for proliferative vitreoretinopathy. Ophthalmology 95(7):865-869. https://doi.org/10.1016/s0161-6420(88)33081-2

27. Lopez R, Chang S (1992) Long-term results of vitrectomy and perfluorocarbon gas for the treatment of severe proliferative vitreoretinopathy. Am J Ophthalmol 113(4):424-428. https://doi.org/ 10.1016/s0002-9394(14)76166-5

28. de Silva DJ, Kwan A, Bunce C, Bainbridge J (2008) Predicting visual outcome following retinectomy for retinal detachment. $\mathrm{Br}$ J Ophthalmol 92(7):954-958. https://doi.org/10.1136/bjo.2007. 131540

29. Shalaby KA (2010) Relaxing retinotomies and retinectomies in the management of retinal detachment with severe proliferative vitreoretinopathy (PVR). Clin Ophthalmol 4:1107-1114. https:// doi.org/10.2147/opth.s4934

30. Stopa M, Kociecki J (2011) Anatomy and function of the macula in patients after retinectomy for retinal detachment complicated by proliferative vitreoretinopathy. Eur J Ophthalmol 21(4):468-472. https://doi.org/10.5301/ejo.2010.6069

31 Aaberg TM (1988) Management of anterior and posterior proliferative vitreoretinopathy. XLV. Edward Jackson memorial lecture. Am J Ophthalmol 106(5):519-532. https://doi.org/10.1016/00029394(88)90580-6

32. Tobalem SJ, Weinberger A, Kropp M, Malcles A, JonescuCuypers C, Souteyrand G, Thumann G (2020) Chorioretinal toxicity of perfluorooctane (Ala Octa): results from 48 surgical procedures in Geneva. Am J Ophthalmol 218:28-39. https://doi. org/10.1016/j.ajo.2020.05.014

Publisher's note Springer Nature remains neutral with regard to jurisdictional claims in published maps and institutional affiliations. 